\title{
JOINT BEAMFORMING AND CHANNEL ESTIMATION FOR PILOT-AIDED WCDMA SYSTEMS
}

\author{
Xavier Mestre, Montse Nájar, Javier R. Fonollosa \\ Department of Signal Theory and Communications \\ Universitat Politècnica de Catalunya \\ c/ Jordi Girona 1-3, Mòdul D-5, Campus Nord UPC \\ 08034 Barcelona (Spain) \\ e-mail:\{mestre,najar,fono\}@gps.tsc.upc.es
}

\begin{abstract}
The problem of joint beamforming and channel estimation for multi-rate multi-code systems is addressed. Usual schemes perform this filtering/estimation operation making use of a training sequence time-multiplexed with the transmitted data. However if pilot and traffic signals are transmitted simultaneously using distinct code allocation -as it is the case in recent standards such as CDMA2000 or WCDMA- these schemes tend to fail. This paper proposes semi-blind techniques to overcome the uplink autointerfering effects of such systems. It is shown that the semi-blind approach yields substantially better performance results thanks to the implicit modeling of the unknown traffic data.
\end{abstract}

\section{INTRODUCTION}

Adaptive antenna arrays have been widely proposed as one of the most attractive means of improving the system coverage and spectral efficiency in high capacity mobile radio networks. The unprecedented growth of wireless markets points to the introduction of novel and cost-effective enabling technologies in the radio link. Among all potential candidates, spatial-temporal processing techniques can certainly help to alleviate congestion problems, boosting the spectrum availability and allowing a proper management of the increasingly high demand for wireless services. This fact has been recognized in most recent standardization efforts for Third Generation Mobile Communication System $^{1}$, which already include explicit features to accommodate smart antennas capabilities [1]. Consequently, as different proposals for a flexible third generation standard converge towards a single definition, the need for tailored receiver structures and algorithms becomes more imperative.

One of the fundamental characteristics of third generation systems is their inherent capability to support conveyance of multiple services at distinct bit rates. The mod-

This work was partially supported by the European Comission under IST Project METRA; the Spanish Govermment (CYCIT) TIC98-0412 TIC98-0703; and the Catalan Government (CIRIT) 1998SGR 00081.

${ }^{1}$ Or UMTS (Unversal Mobile Telecommumications System), as such system is commonly referred to in European contexts. ulation framework harmonizing such transmissions is chosen to be intrinsically flexible with respect to both the number of services simultaneously supported and their corresponding bit rate. This multi-rate adaptability of the system is attained by means of Multi-code access, where High Bit Rate users multiplex their information streams onto multiple codes, and Variable Spreading Length access, where signatures of different lengths are assigned to users with different data rates.

\section{MULTI-RATE MULTI-CODE SIGNAL}

Let us consider the reception of a WCDMA-modulated signal by an antenna array of $P$ elements. In what follows, $r_{p}(t)$ represents the baseband continuous-time signal received by the $p$-th antenna. Assuming that the signal has been transmitted through a frequency-selective channel with impulse response of maximum length $L$ chips, the baseband signal received at sensor $p$ sampled at the chip rate $\left(1 / T_{c}\right)$ can be modeled as follows:

$$
r_{p}\left(n T_{c}\right)=\sum_{l=1}^{L} h_{p}(l, n) x\left((n-l) T_{c}\right)+n_{p}\left(n T_{c}\right)
$$

with $n_{p}\left(n T_{c}\right)$ the noise plus interference component received at sensor $p, x\left(n T_{c}\right)$ the signal transmitted by the user of interest sampled at the chip rate and $h_{p}(l, n)$ the time-varying channel impulse at sensor $p$ including pulseshaping and matched filtering. The mobile station is assumed to map the underlying data sequence to $Q$ distinct and synchronized spreading sequences with the same period $N_{c}$. In the sequel, $Q$ will be referred to as the number of physical channels transmitted by the user of interest, and $N_{c}$ the duration of a spreading period in number of chips. The spreading sequences are denoted $s_{1}(n) \ldots s_{Q}(n)$ with $n=1 \ldots N_{c}$. The number of symbols transported over one period of the $q$-th spreading code is denoted $N_{b}^{q}$, so that the associated spreading factor is variable for each code and equal to $N_{b}^{q} / N_{c}$ for $q=1 \ldots Q$.

The discrete-time transmitted signal can be expressed as:

$$
x\left(n T_{c}\right)=\sum_{m=-\infty}^{\infty} \mathbf{b}^{T}(m) \mathbf{s}\left(n-m N_{c}\right)
$$

with $\mathbf{b}(m)$ a $N_{b} \times 1\left(N_{b}=\sum_{q=1}^{Q} N_{b}^{q}\right)$ vector containing 
the symbols transmitted during the $m$-th spreading period and $\mathbf{s}(n)$ a $N_{b} \times 1$ vector with the segments of spreading sequences associated with each of the symbol intervals. Note that each element of $\mathbf{s}(n)$ has been zero-padded outside the associated symbol interval (see Figure 1). Let us now gather $M=N_{c}+L-1$ samples of the received signal at sensor $p$ into a column vector $\mathbf{r}_{p}$. Figure 2 depicts the structure of this vector in the case $Q=1$. Assuming perfect synchronization with the user of interest, the received signal vector corresponding to the transmission of one spreading period may be expressed as:

$$
\mathbf{r}_{p}=\mathbf{S}\left(\mathbf{b} \otimes \mathbf{h}_{p}\right)+\mathbf{n}_{p}
$$

with $\mathbf{h}_{p}$ a vector containing the channel impulse response at sensor $p, \mathbf{n}_{p}$ the noise plus multi-user interference contribution and $\mathbf{S}$ a $M \times L N_{b}$ matrix containing the distinct convolution matrices constructed from each of the elements of $\mathrm{s}\left(1 \ldots N_{c}\right)$. Note that we have assumed that the channel is approximately stationary within observation intervals of length equal to a spreading period.

Grouping the result in (3) for the $P$ sensors we obtain:

$$
\mathbf{R}=\left[\mathbf{r}_{1} \cdots \mathbf{r}_{P}\right]=\mathbf{S}\left(\mathbf{b} \otimes \mathbf{I}_{L}\right) \mathbf{H}+\mathbf{N}
$$

with $\mathbf{I}_{L}$ the $L \times L$ identity matrix, $\mathbf{H}=\left[\mathbf{h}_{1} \cdots \mathbf{h}_{P}\right]$ the two-dimensional channel matrix and $\mathbf{N}=\left[\mathbf{n}_{1} \cdots \mathbf{n}_{P}\right]$ the spatial-temporal noise plus multi-user interference component, modeled as a circularly symmetric complex zero-mean Gaussian matrix with covariance:

$$
\begin{aligned}
& E\left[\operatorname{vec}[\mathbf{N}] \operatorname{vec}^{H}[\mathbf{N}]\right]=\left(\mathbf{C}_{n}^{s}\right)^{*} \otimes \mathbf{I}_{M} \\
& E\left[\operatorname{vec}[\mathbf{N}] \operatorname{vec}^{T}[\mathbf{N}]\right]=\mathbf{0}
\end{aligned}
$$

Note that we are assuming that the noise plus interference component of the received signal is temporally white, although it has an unknown spatial distribution $\left(\mathbf{C}_{n}^{s}\right)$.

Let us now assume that at each spreading period a set of $N_{k}$ symbols (those corresponding to the Pilot sequence) are known to the receiver, while the other $N_{u}$ convey unknown traffic information $\left(N_{b}=N_{k}+N_{u}\right)$. Thus, we separate the previously defined matrices into two parts corresponding to known and unknown data:

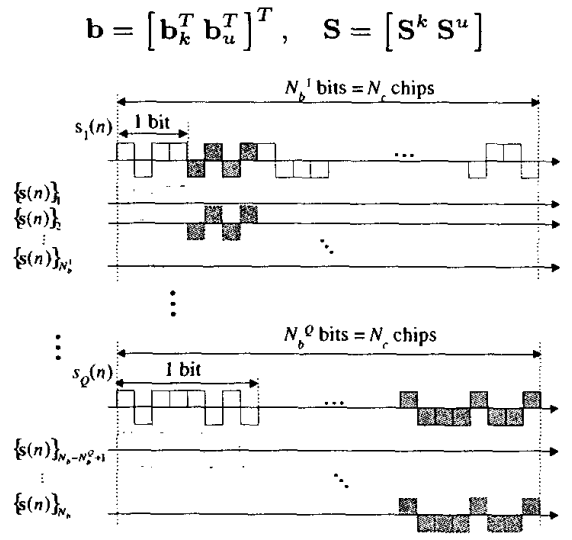

Figure 1: Generation of the signature vector $\mathrm{s}(n)$. The $i$-th element of this vector is denoted $\{\mathbf{s}(n)\}_{i}$.
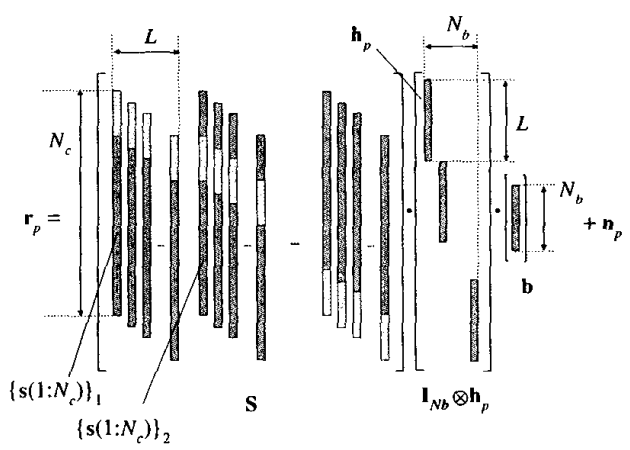

Figure 2: Schematic representation of the received signal vector at sensor $p\left(\mathbf{r}_{p}\right)$ assuming the use of only one spreading code $(Q=1)$.

Note that indexes $k$ and $u$ stand for known and unknown component respectively. With these definitions, the received signal can be expressed as:

$$
\mathbf{R}=\left[\mathbf{S}^{k}\left(\mathbf{b}_{k} \otimes \mathbf{I}_{L}\right)+\mathbf{S}^{u}\left(\mathbf{b}_{u} \otimes \mathbf{I}_{L}\right)\right] \mathbf{H}+\mathbf{N}
$$

\section{PROBLEM STATEMENT}

The scope of the paper is restricted to the reception with a narrowband beamformer. It is clear that, unless the channel matrix $\mathbf{H}$ has rank 1, this is by no means the optimum structure from a maximum likelihood point of view. However, the use of a narrowband beamformer is fully justified by the reduction of the receiver complexity with respect to more general space-time equalizers.

Let $\mathbf{w}$ denote a $P \times 1$ column vector containing the complex weights of the narrowband beamformer. The received signal after the spatial-processing stage can be expressed as:

$$
\mathbf{y}=\mathbf{R} \mathbf{w}=\left[\mathbf{S}^{k}\left(\mathbf{b}_{k} \otimes \mathbf{I}_{L}\right)+\mathbf{S}^{u}\left(\mathbf{b}_{u} \otimes \mathbf{I}_{L}\right)\right] \mathbf{h}+\mathbf{n}
$$

with $\mathbf{h}=\mathbf{H w}$ and $n=\mathbf{N w}$, so that:

$$
E\left[\mathbf{n n}^{H}\right]=\left(\mathbf{w}^{T}\left(\mathbf{C}_{n}^{s}\right)^{*} \mathbf{w}^{*}\right) \mathbf{I}_{M} \equiv \sigma^{2} \mathbf{I}_{M}
$$

Our objective is to design the spatial filter and the channel estimator avoiding the potential auto-interference of the unknown data sequence. To this end, the proposed scheme has to exploit both the known pilot sequence (training-sequence approach) and the knowledge of the statistical structure of the unknown received signal (blind approach). Consequently, a semi-blind approach seems the most indicated solution.

\subsection{Unconditional Semi-blind Approach}

In [2] we proposed a solution to the problem based on a semi-blind Deterministic (Conditional) ML scheme. Here we take another point of view and propose an scheme based on the Unconditional criterion. According to this approach, unknown symbols are regarded as random variables instead of deterministic parameters [3]. If we choose these variables to be zero-mean Gaussian-distributed, the approach is referred to as Gaussian ML (GML). Under this assumption 
the ML cost function to be maximized becomes [4]:

$$
f_{G M L}(\mathbf{y})=\frac{1}{\operatorname{det}^{M}\left(\pi \mathbf{C}_{y}\right)} e^{-\operatorname{tr}\left[\mathbf{C}_{y}^{-1} \overline{\mathbf{c}}_{y}\right]}
$$

with $\tilde{\mathbf{C}}_{y}$ a rank-one estimation of the temporal covariance matrix:

$$
\tilde{\mathbf{C}}_{y}=\left[\mathbf{y}-\mathbf{X}^{k} \mathbf{h}\right]\left[\mathbf{y}-\mathbf{X}^{k} \mathbf{h}\right]^{H}, \quad \mathbf{X}^{k}=\mathbf{S}^{k}\left(\mathbf{b}_{k} \otimes \mathbf{I}_{L}\right)
$$

and $\mathbf{X}^{k}$ the convolution matrix of the known component of the signal. The temporal covariance matrix can be parametrized as follows:

$$
\mathbf{C}_{y}=E\left[\tilde{\mathbf{C}}_{y}\right]=\mathbf{S}_{h}^{u}\left(\mathbf{S}_{h}^{u}\right)^{H}+\sigma^{2} \mathbf{I}, \quad \mathbf{S}_{h}^{u}=\mathbf{S}^{u}\left(\mathbf{I}_{N_{u}} \otimes \mathbf{h}\right)
$$

with $\mathbf{S}_{h}^{u}$ a matrix containing the channel-filtered sequences used to transmit the unknown symbol sequence. Note that we are making the following assumptions regarding the statistical nature of the unknown symbols:

$$
E\left[\mathbf{b}_{u} \mathbf{b}_{u}^{H}\right]=\mathbf{I}, \quad E\left[\mathbf{b}_{u} \mathbf{b}_{u}^{T}\right]=\mathbf{0}, \quad E\left[\mathbf{b}_{u}\right]=\mathbf{0}
$$

The GML solution can be found taking derivatives of the log-likelihood function with respect to the channel impulse response, the noise power and the beamforming weights. The equivalent cost function is taken as:

$$
\eta_{G M L}=\log \operatorname{det}\left(\mathbf{C}_{y}\right)+\operatorname{tr}\left(\mathbf{C}_{y}^{-1} \tilde{\mathbf{C}}_{y}\right)
$$

After easy but somewhat lengthy calculations the derivatives with respect to $\sigma^{2}$ and $\mathbf{h}^{*}$ can be expressed as [5]:

$$
\begin{aligned}
& \frac{\delta \eta_{G M L}}{\delta \sigma^{2}}=\operatorname{tr}\left[\mathbf{C}_{y}^{-1}-\mathbf{C}_{y}^{-1} \tilde{\mathbf{C}}_{y} \mathbf{C}_{y}^{-1}\right] \\
& \frac{\delta \eta_{G M L}}{\delta \mathbf{h}^{*}}=\mathbf{D h}-\left(\mathbf{X}^{k}\right)^{H} \mathbf{C}_{y}^{-1}\left[\mathbf{y}-\mathbf{X}^{k} \mathbf{h}\right]
\end{aligned}
$$

being:

$$
\mathbf{D}=\sum_{i=1}^{N_{u}}\left(\mathbf{S}_{i}^{u}\right)^{H}\left(\mathbf{C}_{y}^{-1}-\mathbf{C}_{y}^{-1} \tilde{\mathbf{C}}_{y} \mathbf{C}_{y}^{-1}\right) \mathbf{S}_{i}^{u}
$$

and $\mathbf{S}_{i}^{u}$ the $M \times L$ fragments of matrix $\mathbf{S}^{u}$ associated with the $i$-th unknown symbol interval:

$$
\mathbf{S}^{u}=\left[\mathbf{S}_{1}^{u} \mathbf{S}_{2}^{u} \cdots \mathbf{S}_{N_{u}}^{u}\right]
$$

An expression for the GML channel estimator can be obtained forcing the gradient in (16) to zero:

$$
\hat{\mathbf{h}}_{G M L}=\left[\hat{\mathbf{D}}+\left(\mathbf{X}^{k}\right)^{H} \hat{\mathbf{C}}_{y}^{-1} \mathbf{X}^{k}\right]^{-1}\left(\mathbf{X}^{k}\right)^{H} \hat{\mathbf{C}}_{y}^{-1} \mathbf{R} \hat{\mathbf{w}}_{G M L}
$$

with $\hat{\mathbf{D}}$ and $\hat{\mathbf{C}}_{y}$ respectively equal to the expression for $\mathbf{D}$ and $\mathbf{C}_{y}$ replacing $\mathbf{h}$ and $\sigma^{2}$ by their GML estimates and $\hat{\mathbf{w}}_{G M L}$ the GML estimator of the beamforming weights. In order to obtain a more enlightening interpretation of the semi-blind GML channel estimator we rearrange the gradient expression in the following way:

$$
\begin{aligned}
\frac{\delta \eta_{G M L}}{\delta \mathbf{h}^{*}}= & \mathbf{C}_{\mathbf{x}} \mathbf{h}-\left(\hat{\mathbf{X}}^{u}\right)^{H} \mathbf{C}_{y}^{-1}\left[\mathbf{y}-E\left[\mathbf{X}^{k}\right] \mathbf{h}\right] \\
& -\left(\mathbf{X}^{k}\right)^{H} \mathbf{C}_{y}^{-1}\left[\mathbf{y}-E\left[\mathbf{X}^{u}\right] \mathbf{h}\right]
\end{aligned}
$$

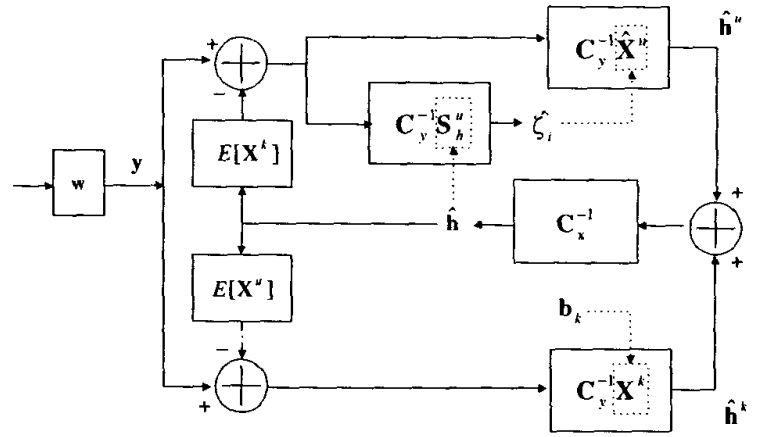

Figure 3: Block diagram of the semi-blind channel estimator/beamforming scheme according to the GML approach.

where $E\left[\mathbf{X}^{k}\right]$ and $E\left[\mathbf{X}^{u}\right]$ stand for the a priori known mean values of $\mathbf{X}^{k}$ and $\mathbf{X}^{u}=\mathbf{S}^{u}\left(\mathbf{b}_{u} \otimes \mathbf{I}_{L}\right)$ (in our case, $E\left[\mathbf{X}^{k}\right]=$ $\mathbf{X}^{k}, E\left[\mathbf{X}^{u}\right]=\mathbf{0}$ ). Matrix $\hat{\mathbf{X}}^{u}$ is defined as a structured estimation of $\mathrm{X}^{u}$ :

$$
\hat{\mathbf{X}}^{u}=\sum_{i=1}^{N_{u}} \zeta_{i} \mathbf{S}_{i}^{u}, \quad \zeta_{i}=\mathbf{h}^{H}\left(\mathbf{S}_{i}^{u}\right)^{H} \mathbf{C}_{y}^{-1}\left[\mathbf{y}-\mathbf{X}^{k} \mathbf{h}\right]
$$

and $\zeta_{i}$ represents the output of a conventional MMSE equalizer in which the known part of the signal has been subtracted from the input. Note that the MMSE equalizer appears inherently in the GML formulation. Finally, the correlation matrix $\mathbf{C}_{\mathbf{x}}=E\left[\mathbf{X}^{H} \mathbf{C}_{y}^{-1} \mathbf{X}\right]$ can be expressed as:

$$
\mathbf{C}_{\mathbf{x}}=\left(\mathbf{X}^{k}\right)^{H} \mathbf{C}_{y}^{-1} \mathbf{X}^{k}+\sum_{i=1}^{N_{u}}\left(\mathbf{S}_{i}^{u}\right)^{H} \mathbf{C}_{y}^{-1} \mathbf{S}_{i}^{u}
$$

In view of (20), the semi-blind GML channel estimation process can be interpreted as shown in Figure 3. In a first stage, the mean values of the known and unknown component of the channel-filtered transmitted signal are subtracted from the input. Each result is then passed through a filter matched to the training sequence $\left(\mathbf{C}_{y}^{-1} \mathbf{X}^{k}\right)$ and the reconstructed unknown signal component $\left(\mathbf{C}_{y}^{-1} \hat{\mathbf{X}}^{u}\right)$ respectively. The output of the two correlators-named $\hat{\mathbf{h}}^{u}$ and $\hat{\mathbf{h}}^{k}$ in Figure 3- are two biased estimations of the channel impulse response. Assuming that the unknown component of the signal has been reconstructed perfectly and that matrix $\mathbf{C}_{y}$ is known, the mean of these estimators can be expressed as:

$$
\begin{aligned}
& E\left[\hat{\mathbf{h}}^{u}\right]=E\left[\left(\mathbf{X}^{u}\right)^{H} \mathbf{C}_{y}^{-1}\left(\mathbf{X}^{u}\right)\right] \mathbf{h} \\
& E\left[\hat{\mathbf{h}}^{k}\right]=\left(\mathbf{X}^{k}\right)^{H} \mathbf{C}_{y}^{-1}\left(\mathbf{X}^{k}\right) \mathbf{h}
\end{aligned}
$$

The final channel estimation is obtained combining the results of both branches before eliminating the resulting bias by means of matrix $\mathbf{C}_{\mathbf{x}}^{-1}$.

Let us now turn to the design of the beamforming weight vector. The gradient of the original cost function with respect to the beamforming weights can be expressed as:

$$
\frac{\delta \eta_{G M L}}{\delta \mathbf{w}^{*}}=\mathbf{R}^{H} \mathbf{C}_{y}^{-1}\left[\mathbf{R} \mathbf{w}-\mathbf{X}^{k} \mathbf{h}\right]
$$

Substituting $\mathbf{h}$ by the estimated channel impulse response in (19) we obtain the GML solution for the spatial filter: 


$$
\begin{gathered}
{\left[\mathbf{R}^{H} \hat{\mathbf{C}}_{y}^{-1} \mathbf{R}\right] \hat{\mathbf{w}}_{G M L}=\gamma_{\min }\left[\mathbf{R}^{H} \mathbf{B R}\right] \hat{\mathbf{w}}_{G M L}} \\
\mathbf{B}=\hat{\mathbf{C}}_{y}^{-1} \mathbf{X}^{k}\left[\left(\mathbf{X}^{k}\right)^{H} \hat{\mathbf{C}}_{y}^{-1} \mathbf{X}^{k}+\hat{\mathbf{D}}\right]^{-1}\left(\mathbf{X}^{k}\right)^{H} \hat{\mathbf{C}}_{y}^{-1}
\end{gathered}
$$

where we are implicitly imposing the constraint $\hat{\mathbf{w}}_{G M L}^{H} \mathbf{R}^{H} \mathbf{B R} \hat{\mathbf{w}}_{G M L}=1$ (unit desired signal power) on the beamforming weight vector.

The GML solution can be obtained solving (15), (19) and (26), following for instance an iterative procedure. Note, however, that when the number of incoming snapshots is high, one may use an asymptotic approximation to the GML criterion suppressing $\hat{\mathbf{D}}$ in (19) and (27).

\subsection{Training-Only Approach}

The classical Training-only scheme can be obtained neglecting the presence of unknown symbols in the signal model. The solution can be expressed as [6]:

$$
\begin{aligned}
\hat{\mathbf{h}}_{T O} & =\left[\left(\mathbf{X}^{k}\right)^{H} \mathbf{X}^{k}\right]^{-1}\left(\mathbf{X}^{k}\right)^{H} \mathbf{R}_{\text {w }}{ }_{T O} \\
{\left[\mathbf{R}^{H} \mathbf{R}\right] \hat{\mathbf{w}}_{T O} } & =\gamma_{\min }\left[\mathbf{R}^{H} \mathbf{P}_{\mathbf{X}^{k}} \mathbf{R}\right] \hat{\mathbf{w}}_{T O}
\end{aligned}
$$

with $\mathbf{P}_{\mathbf{X}^{k}}=\mathbf{X}^{k}\left[\left(\mathbf{X}^{k}\right)^{H} \mathbf{X}^{k}\right]^{-1}\left(\mathbf{X}^{k}\right)^{H}$ and being the new constraint $\hat{\mathbf{w}}_{T O}^{H} \mathbf{R}^{H} \mathbf{P}_{\mathbf{X}^{k}} \mathbf{R} \hat{\mathbf{w}}_{T O}=1$.

\section{SIMULATIONS}

The objective of these simulations is to demonstrate the effectiveness of the semi-blind GML approach in order to overcome the auto-interfering effect of the traffic channel code-multiplexed with the pilot channel. Simulations assumed a linear uniformly spaced array of 4 elements with an inter-element separation of half a wavelength at the carrier frequency $(1950 \mathrm{MHz})$. Three mobile stations transmitted WCDMA-modulated signals (according to current ETSI specifications) consisting of two physical channels $(Q=2)$ : a traffic signal with a spreading factor of 8 chips/bit and a pilot sequence with a repetition period of 256 chips. See [1] for details of the modulation format. The simulation length was set equal to $M=270 \mathrm{chips}$, so that the channel length assumed at the receiver was $L=15$. The spatial distribution of the channel was generated according to a Laplacian Power Angular Spectrum (variance $1 \mathrm{deg}$.) and its impulse response at each sensor was derived from the Vehicular-A channel model as proposed by ETSI.

Figure 4 represents the channel estimation relative MSE for each of the approaches and the corresponding output SINR provided by the spatial filter. These values were obtained averaging the results of 30 independent realizations. It is seen that when the input SNR increases, the performance of the training-only scheme becomes seriously degraded. This behavior is a consequence of the presence of the traffic component, which is seen by the basestation as an interfering source coming from the direction of arrival of the desired user. Although traffic and pilot channels are mapped to distinct orthogonal codes, the presence of the multipath prevents their perfect separation. This way, unless the unknown component of the signal is properly modeled (as it is under the semi-blind approach), the spatial filter tries to null out the contribution of the desired user while at the same time the channel estimator module is unable to extract the proper information from the pilot.
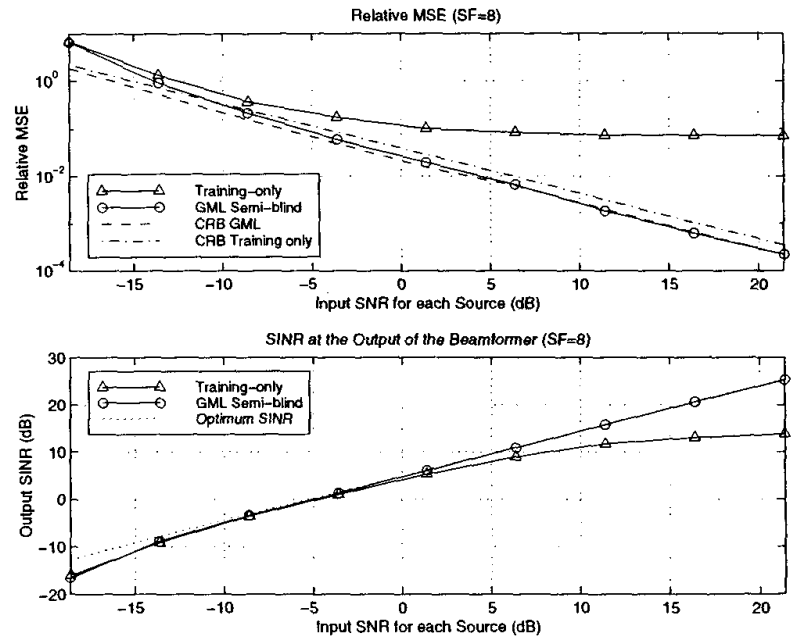

Figure 4: Peformance of the Training-only and the Semiblind approaches.

\section{CONCLUSIONS}

Unconditional semi-blind approaches are proposed in order to overcome the auto-interfering effects of traffic channels on pilot-aided WCDMA systems. In particular, the GML semi-blind channel estimation scheme has been interpreted as an extension of the Training-only case in which the unknown component of the transmitted signal is regenerated by means of a MMSE equalizer. Performance results demonstrate how the semi-blind approach is able to successfully solve the joint beamforming and channel estimation problem, avoiding the degradation caused by the presence of the traffic channel.

\section{REFERENCES}

[1] ETSI, "Evaluation report for ETSI UMTS terrestrial radio access (UTRA) ITU-R RTT candidate," Tech. Rep., ETSI-SMG2, October 1998.

[2] X. Mestre, M. Nájar, C. Antón, and J.R. Fonollosa, "Adaptive beamforming for high bit rate services in the FDD mode of UTRA," in Proc. IEEE VTC (fall), Amsterdam (The Netherlands), Sept. 1999.

[3] P. Stoica and A. Nehorai, "Performance study of conditional and unconditional direction-of-arrival estimation," IEEE Transactions on ASSP, vol. 38, no. 10, pp. 1783-1795, Oct. 1990.

[4] E. DeCarvalho and D. Slock, "Cramer-Rao bounds for semi-blind, blind a training sequence-based channel estimation," in Proc. IEEE SP Workshop on SPAWC, Paris (France), Apr. 1997, pp. 129-132.

[5] X. Mestre, M. Najar, and J.R. Fonollosa, "Joint spatial processing and channel estimation in WCDMA," Submitted to IEEE JSAC, 1999.

[6] M.A. Lagunas, J. Vidal, and A.I. Pérez-Neira, "Joint array combining and MLSE for single-user receivers in multipath gaussian multiuser channels," Submitted to the IEEE JSAC, 1999. 